\title{
On Active Current Selection for Lagrangian Profilers
}

\author{
J. Jouffroy ${ }^{1}$ Q.-Y- Zhou $^{1}$ O. Zielinski ${ }^{2}$ \\ ${ }^{1}$ Mads Clausen Institute, University of Southern Denmark, DK-6400 Sønderborg, Denmark. E-mail: \\ $\{$ jerome, qiz\}@mci.sdu.dk \\ ${ }^{2}$ Institute for Chemistry and Biology in the Marine Environment (ICBM), University of Oldenburg, Germany. \\ E-mail: oliver.zielinski@univ-oldenburg.de
}

\begin{abstract}
Autonomous Lagrangian profilers are now widely used as measurement and monitoring platforms, notably in observation programs as Argo. In a typical mode of operation, the profilers drift passively at their parking depth before making a vertical profile to go back to the surface. This paper presents simple and computationally-efficient control strategies to actively select and use ocean currents so that a profiler can autonomously reach a desired destination. After briefly presenting a typical profiler and possible mechanical modifications for a coastal environment, we introduce simple mathematical models for the profiler and the currents it will use. We then present simple feedback controllers that, using the direction of the currents and taking into account the configuration of the environment (coastal or deep-sea), is able to steer the profiler to any desired horizontal location. To illustrate the approach, a few results are presented using both simulated currents and real current velocity profiles from the North Sea.
\end{abstract}

Keywords: Lagrangian profilers, underwater vehicles, underactuated systems, sector-of-sight controllers, ocean currents.

\section{Introduction}

Monitoring the oceans is a matter of primary importance, not only for ongoing research related to global warming, but also for other activities such as oceanographic studies, the fishing industry, and military operations. For many of them, a number of different parameters such as temperature and salinity are regularly measured over time and at different locations around the globe. Among the means available to obtain these measurements, autonomous Lagrangian profilers such as the ALACE (Davis et al., 1992), the PROVOR (Loaec et al., 1998) or the NEMO floats (see Figure 1) have been widely used in different observation programs such as the Argo Project (Freeland et al., 2009).

Typically, a profiling drifter, or simply called float, takes measurements at different depths along vertical profiles by controlling its depth with an external bladder connected to a hydraulic pump. In open waters, drifters are also used to measure horizontal velocities of subsurface ocean currents by "parking" the drifter at a depth where the current is located, letting it drift passively along with it, and then compute the current velocity from the distance between the location of the initial dive and the resurfacing of the drifter a few days later (Roemmich et al., 2004). This mode of transport is also very energy-efficient, as the profilers can travel great distances at a low energy cost, only using the power provided by batteries to change their depths, until these batteries eventually run out and the profilers are lost at sea.

An important part of the cost of operating profiling floats is coming from deployment from ships, since 


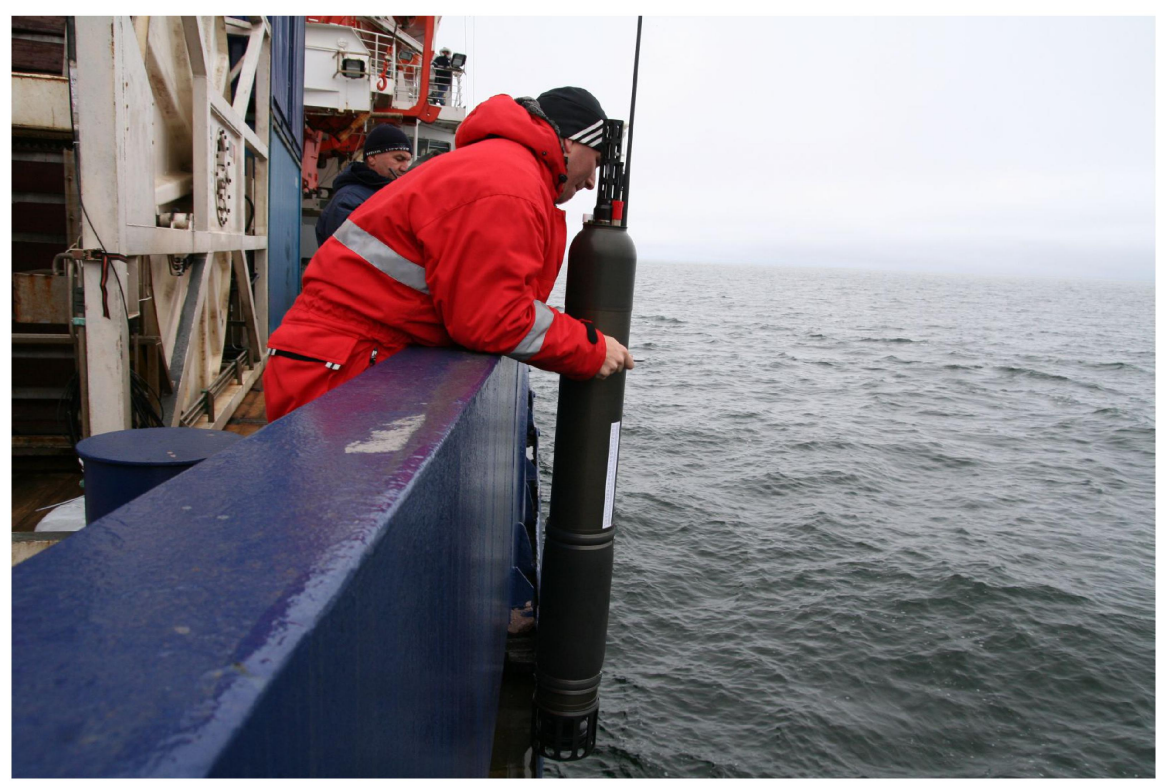

Figure 1: Launching of a NEMO profiler (courtesy of OPTIMARE).

the profilers typically need to be launched at specific locations. In order to allow for more flexibility in deployment, different types of missions, as well as possible recovery for reusability, this paper proposes control strategies for profilers so that they are able to autonomously "piggy-back" ocean currents to achieve horizontal displacement. Specifically, we go one step further than the above-mentioned passive mode of transport whereby the profiler will actively select a current, or park at the depth where it is located, provided the current lies in the general direction of the desired destination. Our feedback controllers ensure that, using the knowledge of the current direction combined with the profiler horizontal position, the profiler will eventually reach a neighborhood of any desired destination, provided a few mild but realistic assumptions are made on the structure of the currents.

The rest of this paper is organized as follows. After this introduction, we give in section 2 a brief description of a typical profiler, with a focus on the external bladder responsible for vertical motion. We will also consider mechanisms that, in a coastal environment scenario, will allow the profiler to stay put on a relatively shallow sea floor until the direction of the tidal current is considered as acceptable. Then, we will introduce a simple mathematical description of the system (i.e. the profiler and the currents) in order to represent its 3 Degrees of Freedom (DOF) motion. Section 3 will be dedicated to the feedback controllers themselves, each one corresponding to a particular environment. We first propose a feedback controller for a drifter evolving in a coastal environment, i.e. with relatively shallow depths, where oscillating tidal currents are tapped for transport, similarly to energy-efficient locomotion strategies used by some marine animals, grouped under the term Selective Tidal-Stream Transport (see Forward and Tankersley (2001)). We show mathematically that the system in closed-loop, i.e. the profiler controlled by the proposed algorithm, will converge in finite time to a finite region around the chosen destination point. Our second scenario considers a deep-sea environment with several layers, where each current layer has a different direction. To illustrate the potential of our approach, test results of a simulation of our system using actual current velocity profiles from the North Sea are briefly discussed in Section 4. Finally, a few concluding remarks end the paper. Earlier versions of some parts of the present paper appeared in Jouffroy et al. (2011).

\section{Profiler description and mathematical modeling}

Roughly speaking, an autonomous Lagrangian profiler is a float whose buoyancy can be adjusted, principally by using an external bladder situated at the bottom of 


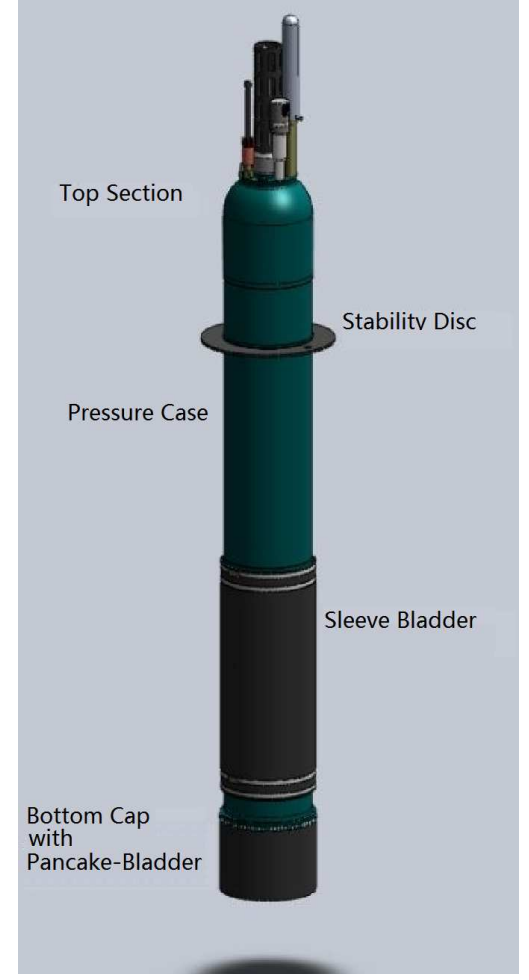

Figure 2: Schematic of a NEMO float (courtesy of OPTIMARE).

the profiler (see Figure 2). This bladder is inflated with hydraulic fluid coming from a pump, thus increasing volume and buoyancy, resulting in turn in the vehicle going upwards. Inversely, a decrease in volume results in the drifter diving until it reaches a depth where it is neutrally buoyant again.

Since the vehicle is not actuated horizontally, its planar motion is only driven by horizontal ocean currents. In the context of a deep-sea environment, several layers of relatively constant currents with different magnitude and direction can be present, each layer depending on the depth. Hence, horizontal motion towards a destination can simply achieved, with the profiler parking at the right depth and use the current as a kind of conveyor belt. In a coastal scenario however, and with relatively shallow depths of up to 100-200 meters, the means of transportation consists mostly of one layer of oscillating and therefore time-varying tidal currents, which result in the profiler going around in circles, or in horizontal corkscrew-like trajectories if the tidal currents are superposed with a constant main current.

In this case, a simple idea is to use the seabed as a means to slow down, or even stop the profiler when the direction of the oscillating current is not satisfactory. Two possible mechanisms can be used in this regard.
The first one, proposed in André et al. (2010), consists of anti-drift clamp or claw-like pikes placed at the bottom of the profiler (see illustration of the "clamp model" in Figure 3) that will allow the latter to cling to the seabed and stop entirely. However, such a mechanism might lose its efficiency in situations where the seabed soil is too hard for the clamps to grip. Hence, as an alternative, a second solution is a simple device appended to the profiler to significantly increase friction with the seabed-and therefore slow down the horizontal motion of the profiler. The device could consist of a simple weight attached to the profiler with a rope, or one or several chains with pikes to increase friction further (this "weight model" is also represented in Figure $3)$.

In order to try different scenarios (coastal and deepsea), a simulator was designed using a mathematical model of the dynamics of a profiler subject to horizontal currents. When the profiler evolves freely in open waters (i.e. without any contact with a seabed), a simple 3DOF translational model can be expressed as

$$
\begin{aligned}
m_{x} \ddot{x}+d_{x}\left(\dot{x}-u_{c}(z, t)\right) & =0 \\
m_{y} \ddot{y}+d_{y}\left(\dot{y}-v_{c}(z, t)\right) & =0 \\
m_{z} \ddot{z}+d_{z} \dot{z} & =G(t)
\end{aligned}
$$

where $x, y, z$ are standard notations representing the north-east-down coordinates, represented in Figure 3 (see also Fossen (2002)), $m_{x}, m_{y}, m_{z}$ are inertia parameters, while $d_{x}, d_{y}, d_{z}$ account for the damping of the system. Depth and time-dependent signals $u_{c}(z, t)$ and $v_{c}(z, t)$ represent the horizontal ocean currents, which will be specified later, according to whether the profiler is in a coastal or deep-sea scenario. Note that in the above model, we assume no vertical current. Variable $G(t)$ is the force created by the added effects of gravity, buoyancy, including the one for the actuated bladder. Obviously, the amplitude of the force determines the profiler going up or down.

In the coastal scenario, the dynamics of the profiler motion will obviously change upon reaching the seabed, also depending on which braking mechanism is used. Indeed, for the clamp/claw mechanism, eq. (1) and (2) are changed into zero velocities, while (3) is replaced with

$$
m_{z} \ddot{z}+d_{z} \dot{z}=G(t)+F_{\text {seabed }}(t), \dot{z}(0)=0
$$

where $F_{\text {seabed }}(t)$ represents the interaction between the clamp and the seabed. When $G(t)$ is positive, $F_{\text {seabed }}(t)$ is negative and equal in magnitude, while if $G(t)$ is negative, $F_{\text {seabed }}(t)$ will act as a stiction represented by the clamp mechanism, i.e. the magnitude of $G(t)$ should be increased until it overcomes the maximum amplitude of $F_{\text {seabed }}(t)$ when the latter is positive. The force $F_{\text {seabed }}(t)$ disappears as $z(t)$ decreases 


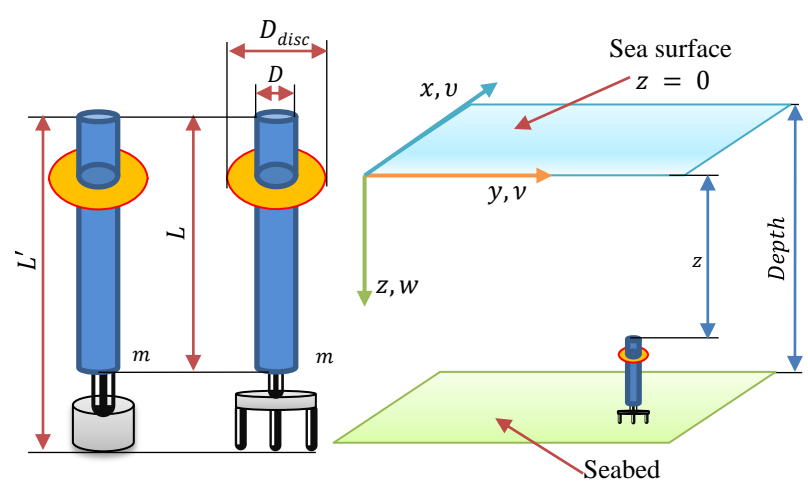

Figure 3: Profiler "braking" mechanisms and coordinates.

again and the profiler leaves the seabed, and the model changes back to (3).

For the so-called weight mechanism model, the dynamics in $z$ while on the seabed are similar. However, the horizontal velocities do not change so abruptly. Instead, additional Coulomb friction forces $f_{x}$ and $f_{y}$ appear on the right-hand side of (1) and (2) as the mechanism touches the seabed.

For the ocean currents in a coastal environment, we opted for a simple time-varying model to represent the corkscrew-like horizontal trajectories followed by profilers, although more complex tidal current model structures can be used (see for example Walters (1987)). Indeed, and similarly to Hill (1994), $u_{c}(z, t)$ and $v_{c}(z, t)$ are written as

$$
\begin{aligned}
& u_{c}(z, t)=u_{c}(t)=u_{0}+C \cos (2 \pi f . t) \\
& v_{c}(z, t)=v_{c}(t)=v_{0}+C \sin (2 \pi f . t)
\end{aligned}
$$

where $u_{0}, v_{0}$ represent a mean current, while $C$ stands for the amplitude of the tidal current oscillation. If this current could be seen as being too regular, not taking into account spatial and time variations, note however that, as will be seen in the next section, the assumptions we used for proving the stability of our controller are valid for a much wider class of time-varying currents.

For the deep-sea case, the currents, that we assume to be stationary and write $u_{c}(z)$ and $v_{c}(z)$, are simply modeled as if-then-else conditions, in which the depth decides on the magnitude and direction of the current.

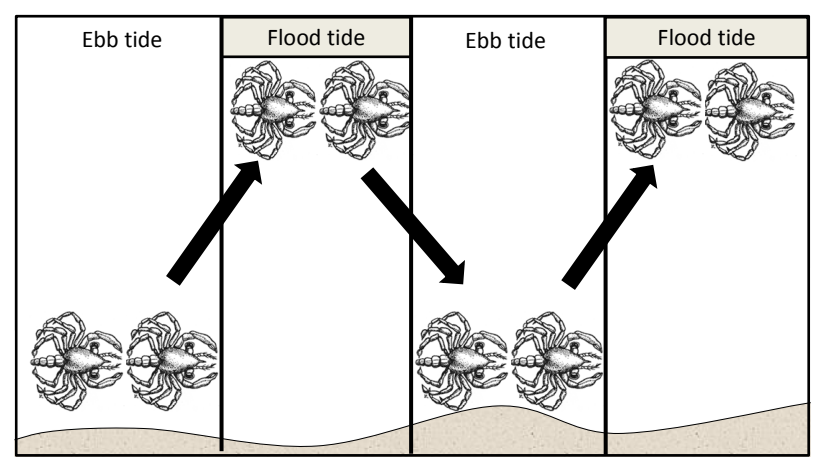

Figure 4: Diagram of blue crab post-larvae migration using Selective Tidal-Stream Transport.

\section{Sector-of-sight feedback controllers}

\subsection{Coastal scenario}

Interestingly, a number of marine animals actively use tidal currents to spread or migrate to different locations, such as spawning areas or feeding grounds. In marine biology, such strategies are referred to as Selective Tidal-Stream Transport, or STST (see Forward and Tankersley (2001); Gibson (2003)). For example, blue crab post-larvae swim up in the water column at flooding tide in order to go upstream in estuaries (see Welch and Forward (1978) and Figure 4). Using cues such as salinity and turbulence, they stay in the water column until the current speed decreases and then start descending to the bottom. As another example, the adult plaice, living on continental shelves, migrate to feeding areas after spawning by traveling in a specific direction using tidal transport (Forward and Tankersley, 2001). In some sense, it can then be argued that our control algorithm captures the same behavior as the plaice's migration strategy. In Weihs (1978), it is shown that tidal stream transport is energetically more efficient than constant swimming with, in some cases, up to $90 \%$ in energy savings.

In order to save on computer power, thereby saving energy further, our feedback algorithm should be as simple as possible for the embedded computer. Thus, for control design, we will use a discrete-time system representing the dynamics of a Lagrangian profiler with a very low sampling frequency. The motion we are interested in being linked with the period of tides (around 12 hours), a sampling period of several minutes is sufficient. This also allows for a low consumption for computation on the embedded computer, which can be in sleep mode most of the time. Finally, it can easily be shown that the mathematical model of 
section 2 can be approximated by the following system

$$
X(k+1)=X(k)+X_{c}(k) \cdot u(k)
$$

where $X(k)$ represents the horizontal position of the profiler at a sampled instant, and $X_{c}(k)$ is the incremental displacement induced by the current between two iterations when the profiler is evolving on the surface. Variable $u(k)$ is a control input taking values 0 or 1 , and representing the depth of the profiler. If $u=0$, the profiler is on the seabed, $u=1$ means the profiler is moving freely at the surface. In eq. (7), $X(k)$ and $X_{c}(k)$ are horizontal vectors and are represented by complex numbers, i.e. for example $X(k)=x(k)+i . y(k)$ for the profiler position (see also Jouffroy and Opderbecke (2007) for a similar use of a complex setting in underwater navigation).

On a more control-theoretic perspective, note that it is the very presence of disturbances, as represented by the currents, that allows for the system (7) to be controllable. In this respect, we can therefore infer that despite its relative simplicity, model (7) represents the behavior of a profiler accurately enough for our purposes.

As alluded to in the introduction, the main and simple idea for the controller is that if the tidal current direction lies within the general direction of the destination (which we first assume to be the origin), then we let the float surface and let it be carried away by the current. Otherwise, it should stay put and wait for a better opportunity. More specifically, we define "general direction" as a sector around the line-of-sight (LOS) (see Healey and Lienard (1993) and Fossen et al. (2003) for references on control using the line-of-sight concept) between the actual horizontal position of the profiler and the destination point. This sector, of center $X(k)$ and central angle $2 \theta_{L}$, will in the following be referred to as sector-of-sight. Hence our stabilizing controller based on the sector-of-sight concept is expressed by the following algorithm

$$
\begin{aligned}
& \theta_{\text {los }}(k)=\arg (-X(k)) \\
& \theta(k)=\arg \left(e^{-i \theta_{\text {los }}(k)} \cdot X_{c}(k)\right) \\
& \text { if }-\theta_{L} \leq \theta(k) \leq \theta_{L} \text { then } \\
& \quad u(k)=1 \\
& \text { else } \\
& \qquad u(k)=0 \\
& \text { end }
\end{aligned}
$$

where $\theta_{L}$ is a constant belonging to the interval ]0, $\pi / 3$ ] If the above algorithm will make the profiler aim for the origin of the horizontal plane, note that it can easily be modified to accommodate other target/destination points $X_{T}$ by replacing eq. (8) with

$$
\theta_{\text {los }}(k)=\arg \left(X_{T}-X(k)\right)
$$

Without loss of generality, and for the sake of clarity, we will however assume that $X_{T}=0$.

We will now proceed to proving that with this simple algorithm, the closed-loop system converges in finite time to a neighborhood around the origin. However, it is intuitively clear that the size of this neighborhood, as well, as the time it will take the profiler to reach it will depend on the amplitude and other mathematical properties such as direction, frequency of the tidal currents.

In order to account for as many tidal current variations as possible, as well as allow the possibility for the profiler to travel in all directions, we define a few (mild) restrictions on the current evolution. These are gathered in the Assumption below.

Assumption 1 Let $R$ be a strictly positive constant. For all $k \geq k_{0}$, current variable $X_{c}(k)$ is upper bounded, i.e.

$$
\left\|X_{c}(k)\right\| \leq \bar{r}
$$

where $\bar{r}$ is a constant such that $0<\bar{r}<R$. Furthermore, there exist a constant $T \in \mathbb{N}$ and at least one instant $k^{\prime} \in[k, k+T]$, such that, for all $k \geq k_{0}$, the following inequalities are verified

$$
-\theta_{L} \leq \theta\left(k^{\prime}\right) \leq \theta_{L}
$$

and

$$
\underline{r} \leq\left\|X_{c}\left(k^{\prime}\right)\right\|
$$

where $\underline{r}$ is a constant such that $0<\underline{r} \leq \bar{r}$.

Inequalities (12) and (13) ensure that when the current has the general direction of the target point (in our case, the origin of the plane), its amplitude is sufficient to make it progress toward the target at reasonable velocity. Inequality (11), requiring that any current vector is upper bounded by a constant value, is very mild and easy to satisfy.

Note that Assumption 1 allows to have currents that are more general in their description than usuallydescribed tidal current approximations (see for example eq. (5)-(6) or Hill (1994)), thus taking into account erratic behaviors or uncertainties in the current direction and amplitude.

We are now ready to state the following result proving the convergent behavior of sector-of-sight feedback controller (8)-(10). Note that because of the constraints imposed by the oscillatory nature of tidal currents, the proof can be seen as having an underlying "averaging" tone, and some parts are indeed close in spirit to some studies on time-varying nonlinear systems (see for example Aeyels and Peuteman (1998); Teel et al. (1999)). 
Theorem 1 Under Assumption 1, for all $X\left(k_{0}\right) \in \mathbb{R}^{2}$ (or $\mathbb{C}$ ), there exists a time $k_{1} \geq T$ such that the solution $X(k)$ of closed-loop system (7)-(10) verifies the following inequalities

$$
\|X(k)\| \leq\left\|X\left(k_{0}\right)\right\|+\bar{g}\left(\frac{k-k_{0}-T}{T+1}\right), \forall k \mid k_{0}+T \leq k \leq k_{1}
$$

$$
\|X(k)\| \leq R, \forall k \geq k_{1}
$$

where $\bar{g}$ is a strictly negative and real constant.

Proof. We begin by proving that if $\left\|X\left(k_{1}\right)\right\| \leq R$, then $\|X(k)\| \leq R$ for all $k \geq k_{1}$. If current $X_{c}(k)$ is not in the "right" direction, then $u(k)=0$ and

$$
X(k+1)=X(k)
$$

If sector condition (10) is verified, then

$$
X(k+1)=X(k)+X_{c}(k)
$$

Since, from the controller algorithm equations (8)-(10), $\theta(k)$ is the angle between vectors $-X(k)$ and $X_{c}(k)$, then application of the law of cosines gives

$$
\begin{aligned}
\|X(k+1)\|^{2}= & \|X(k)\|^{2}+\left\|X_{c}(k)\right\|^{2} \\
& -2\|X(k)\| \cdot\left\|X_{c}(k)\right\| \cos \theta(k)(1)
\end{aligned}
$$

Since, from algorithm (8)-(10), $-\pi / 3 \leq \theta(k) \leq \pi / 3$, then $1 / 2 \leq \cos \theta(k)$, and eq. (17) transforms into the following inequality

$$
\|X(k+1)\|^{2} \leq\|X(k)\|^{2}+\left\|X_{c}(k)\right\|^{2}-\|X(k)\| \cdot\left\|X_{c}(k)\right\|
$$

Then, rewrite the right-hand side term of (18) as

$$
f(\alpha, \beta):=\alpha^{2}+\beta^{2}-\alpha \beta
$$

where $\alpha:=\|X(k)\|$ and $\beta:=\left\|X_{c}(k)\right\|$. Using basic results from multivariable calculus, we can see that for all $\alpha, \beta \in[0, R]$, we have

$$
f(\alpha, \beta) \leq R^{2}
$$

Hence, using (18) into (20) and considering Assumption 1 , we have that

$$
\|X(k+1)\| \leq R
$$

when $\|X(k)\| \leq R$ and $\underline{r} \leq\left\|X_{c}(k)\right\| \leq \bar{r}$. Finally, considering (16) and (21), and assuming that $\left\|X\left(k_{1}\right)\right\| \leq R$, (15) is proven by induction. We are now turning our attention to proving inequality (14). To do so, first define the function $g(\cdot, \cdot)$ as

$$
g(\alpha, \beta)=\sqrt{f(\alpha, \beta)}-\alpha
$$

for which we assume this time that $\alpha \in[R,+\infty[$ and $\beta \in[\underline{r}, \bar{r}]$. A simple computation on the derivative of $g$ with respect to $\alpha$ shows that $g(\alpha, \beta)$ is strictly decreasing in $\alpha \in[R,+\infty$ [, for all fixed $\beta \in[\underline{r}, \bar{r}]$. Hence the maximum of $g$ lies on the curve

$$
g(R, \beta)=\sqrt{R^{2}+\beta^{2}-R \beta}-R
$$

Since the term under the square root is an "upward" parabola in $\beta$, the maximum of function $g(R, \beta)$ will be on the limits of interval $[\underline{r}, \bar{r}]$, which in turn implies that the maximum $\bar{g}$ of $g(\alpha, \beta)$ is expressed as

$$
\bar{g}=\sqrt{\max \left(\underline{r}^{2}-\underline{r} R, \bar{r}^{2}-\bar{r} R\right)+R^{2}}-R
$$

It can easily be proven that $\beta^{2}-R \beta$ is strictly negative for all $\beta \in[\underline{r}, \bar{r}]$. Hence $\bar{g}$ is a strictly negative constant. From (18)-(19), when there is a current in the right direction, we have

$$
\left\|X\left(k^{\prime}+1\right)\right\| \leq \sqrt{f\left(\left\|X\left(k^{\prime}\right)\right\|,\left\|X_{c}\left(k^{\prime}\right)\right\|\right)}
$$

which, using (22) and bound (24), leads to

$$
\left\|X\left(k^{\prime}+1\right)\right\|-\left\|X\left(k^{\prime}\right)\right\| \leq \bar{g}
$$

Hence, when $\left\|X\left(k^{\prime}\right)\right\|>R$, it is decreasing at least once on the interval $[k, k+T]$, according to Assumption 1 . This means that we have

$$
\left\|X\left(k_{0}+T+1\right)\right\| \leq\left\|X\left(k_{0}\right)\right\|+\bar{g}
$$

on the interval $\left[k_{0}+T+1, k_{0}+2 T+1\right]$, and, assuming only one instant with the current in the right direction, we have, for the last instant on this interval,

$$
\left\|X\left(k_{0}+2 T+1\right)\right\| \leq\left\|X\left(k_{0}\right)\right\|+\bar{g}
$$

Following the same reasoning, we hence obtain

$$
\left\|X\left(k_{0}+l(T+1)+T\right)\right\| \leq\left\|X\left(k_{0}\right)\right\|+l \bar{g}
$$

for the last instant of interval $\left[k_{0}+l(T+1), k_{0}+l(T+\right.$ $1)+T]$, with $l \in \mathbb{N}^{*}$. On the following interval, we obviously obtain

$$
\left\|X\left(k_{0}+l(T+1)+T+T+1\right)\right\| \leq\left\|X\left(k_{0}\right)\right\|+l \bar{g}+\bar{g}
$$

Now, by proceeding to a simple interpolation-like method for instants between $k_{0}+l(T+1)+T$ and $k_{0}+l(T+1)+T+T+1$ for (26) and (27), we have the new inequality

$$
\left\|X\left(k_{0}+l(T+1)+T+j\right)\right\| \leq\left\|X\left(k_{0}\right)\right\|+l \bar{g}+\frac{j}{T+1} \bar{g}
$$

where $j \in \mathbb{N}$. One can check that when $j=0$ we obtain (26), while when $j=T+1$, we have (27). Finally, 


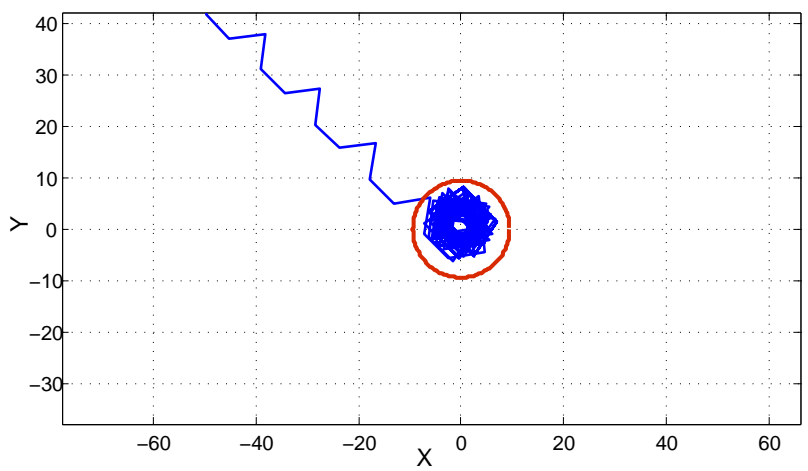

Figure 5: Discrete-time simulation illustrating the convergence behavior of Theorem 1 .

proceeding to the change of variable $k:=k_{0}+l(T+$ $1)+T+j$, eq. (28) is changed into (14). It remains to prove the existence of time $k_{1}$, beyond which $X(k)$ will stay in a ball of radius $R$ around the origin. To do so, use inequality (14) to define

$$
v(k):=\left\|X\left(k_{0}\right)\right\|+\bar{g}\left(\frac{k-k_{0}+T}{T+1}\right), \forall k \geq k_{0}+T
$$

and obtain the "theoretic" time $k_{c}$ at which we have $v\left(k_{c}\right)=R$, which is given by

$$
k_{c}=\frac{T+1}{|\bar{g}|}\left(\left\|X\left(k_{0}\right)\right\|-R\right)+k_{0}+T
$$

However, since $k_{c}$ is real and $\|X(k)\| \leq v(k)$, and estimate of $k_{1}$ can be written as

$$
k_{1} \leq\left\lceil k_{c}\right\rceil
$$

where $[\cdot]$ is the ceiling function. Note that if $\left\|X\left(k_{0}\right)\right\| \leq R$, we simply have $k_{1}=k_{0}+T$. Hence, inequality (29) shows that $X(k)$ converges to the ball of radius $R$ around the origin in finite time.

To illustrate our approach, we simulated discretetime dynamics (7), together with sector-of-sight controller (8)-(10), with $X(0)=-100+i .100, \theta_{L}=\pi / 3$, $X_{T}=0$, and

$$
X_{c}(k)=(-1+8 \cos (k \cdot \pi / 4))+i \cdot(1+8 \sin (k . \pi / 4))
$$

Equation (30) implies that we have the bounds $\underline{r}=$ $8-\sqrt{2}$ and $\bar{r}=8+\sqrt{2}$ required to fulfill Assumption 1 . The result of the simulation is shown in Figure 5, where it can be seen that the horizontal trajectory $X(k)$ of the profiler (in blue) converges to a circle of radius $R>$ $8+\sqrt{2}$ (in red) and eventually stays there, according to Theorem 1 .

\subsection{Deep-sea scenario}

As an extension to the above algorithm, it is also possible to consider other kinds of scenarios. Of particular importance are deep-sea cases where several superposed layers of currents co-exist in the environment. In this situation, the currents are generally of constant amplitude and direction.

In the following, we hence consider the scenario of a profiler navigating into an ocean with three layers of currents, for which we approximate model (1)-(2) as

$$
X(k+1)=X(k)+X_{c}(u(k))
$$

where $X(k)$ and $X_{c}(\cdot)$ still represent the profiler's horizontal position and the current-induced incremental displacement, respectively. However, compared to the coastal scenario and eq. $(7), X_{c}(\cdot)$ is this time a function of $u(k)$, which represents the depth of the profiler, or more accurately the label of each current layer. Hence control input $u(k)$ will take the values $\{1,2,3\}$, while $X_{c}(\cdot)$ is expressed as

$$
X_{c}(u)=\left\{\begin{array}{c}
X_{c 1} \text { if } u=1 \\
X_{c 2} \text { if } u=2 \\
X_{c 3} \text { if } u=3
\end{array}\right.
$$

where $X_{c 1}, X_{c 2}$ and $X_{c 3}$ are constant vectors such that they span the plane positively, i.e. any point in the plane can be expressed as $\alpha_{1} X_{c 1}+\alpha_{2} X_{c 2}+\alpha_{3} X_{c 3}$, where $\alpha_{1}, \alpha_{2}, \alpha_{3}$ are positive numbers.

The idea behind designing a feedback controller steering the profiler to the origin can be presented in a way quite similar to the coastal scenario. Indeed, we check whether each current vector $X_{c i}$ belongs or not to a sector-of-sight. However, since several vectors might fulfill this condition, a logic is needed to select only one vector, resulting in the profiler going to a particular current layer. Hence, a stabilizing controller for the deep-sea scenario would be

$$
\begin{aligned}
& \theta_{\text {los }}(k)=\arg (-X(k)) \\
& \theta_{1}(k)=\arg \left(e^{-i \theta_{\text {los }}(k)} \cdot X_{c 1}(k)\right) \\
& \theta_{2}(k)=\arg \left(e^{-i \theta_{\text {los }}(k)} \cdot X_{c 2}(k)\right) \\
& \theta_{3}(k)=\arg \left(e^{-i \theta_{\text {los }}(k)} \cdot X_{c 3}(k)\right) \\
& \text { if }-\theta_{L} \leq \theta_{1}(k) \leq \theta_{L} \text { then } \\
& \quad u(k)=1 \\
& \text { else if }-\theta_{L} \leq \theta_{2}(k) \leq \theta_{L} \text { then } \\
& \quad u(k)=2 \\
& \text { else } \\
& \quad u(k)=3 \\
& \text { end }
\end{aligned}
$$


where the logic for choosing only one vector in this algorithm simply consists in taking the first vector belonging to the sector-of-sight, as expressed in if-thenelse condition (37)-(38). Note that it is obviously possible to define other ways to choose the current layer used by the profiler. For example, one could also take the vector $X_{c i}$ whose heading is the closest to the lineof-sight. Note that, in this case and because of the assumption on the currents spanning the plane positively, one could totally dispense with checking whether these vectors belong to the sector-of-sight. Indeed, after computing the angles of these vectors with respect to the line-of-sight as in eq. (33)-(36), if-then-else condition (37)-(38) is replaced with

$$
\begin{aligned}
\Theta & =\left[\left|\theta_{1}\right|,\left|\theta_{2}\right|,\left|\theta_{3}\right|\right]^{T} \\
u & =\arg \min (\Theta)
\end{aligned}
$$

However, the main idea of using currents that are in the general direction of the destination remains the same. Using a reasoning similar to the one used in Theorem 1 , it is possible to show that the above algorithm makes the system converge in finite time to a neighborhood around the origin.

Finally, note that the sector-of-sight perspective allows for many extensions/modifications. As an example, consider the fact that algorithm (33)-(36)-(39)-(40) might, under certain conditions, lead to excessive energy spending due to rapid changes of layer, which is indeed possible at each iteration. Then, introducing a memory effect in the form of a unit delay, allows the previously chosen current later to be kept as the direction of transport, until it is not acceptable anymore. A simulation result of this controller with memory effect is shown in Figure 6, with current layers $X_{c 1}=$ $2 i+2, X_{c 2}=-2 i$ and $X_{c 3}=2 i$. All trajectories converge to the origin, with a relatively low number of course changes, corresponding to less changes of depth.

\section{Redeployment scenario in a coastal environment}

The full hybrid model (1)-(4) and the sector-of-sight feedback controller (8)-(10) were also tested using actual current data (direction and strength) from the North Sea. The data was measured with an AADI RCM-9-LW current meter, located at the test area ODAS $\left(\mathrm{N} 54^{\circ} 59^{\prime} 50^{\prime \prime}, \mathrm{E} 7^{\circ} 54^{\prime} 30^{\prime \prime}\right)$, situated at 16 nautical miles west of the island of Sylt in Germany. The current meter was placed at $8 \mathrm{~m}$ above the seabed, or approximatively $15 \mathrm{~m}$ of water depth at low tide level, and recorded data every 10 minutes from March to October 2006. Figure 7 shows the tidal current distribution and evolution over 5 days. Although the current

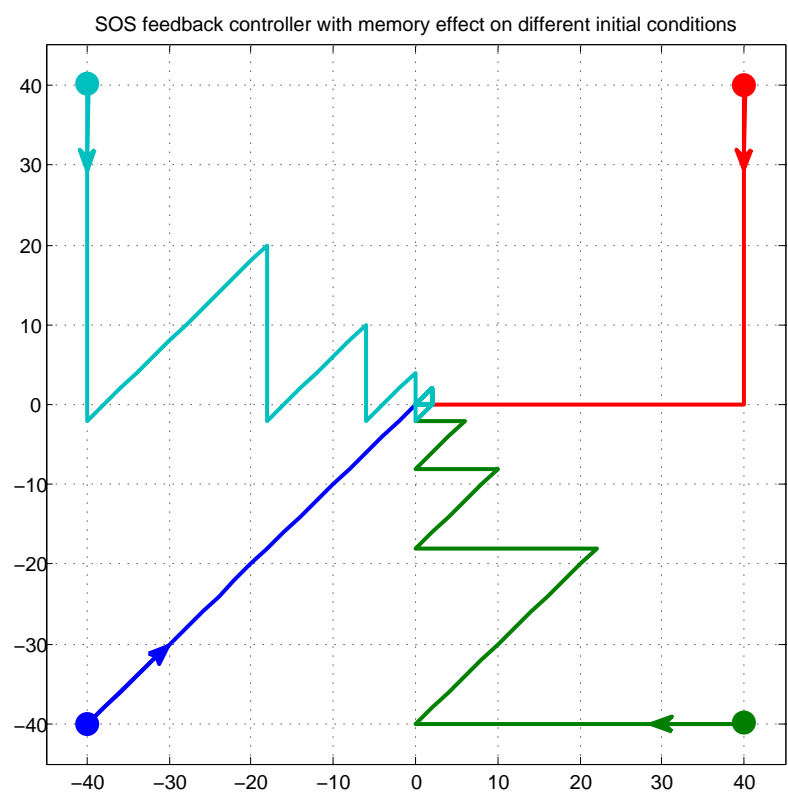

Figure 6: Simulation results of the sector-of-sight feedback controller for deep-sea scenario, with memory effect.

samples seem to be distributed around the origin, an integration of these show a mean current with an amplitude of $3.2 \mathrm{~cm} / \mathrm{s}$, with a mean direction indicated by the black arrow.

In order to test the behavior of the feedback controller, we simulated a scenario whereby the profiler starts from a specific location (i.e. where it was assumed to be first deployed) before transitioning or redeploying itself to two new waypoints before going back to its initial location. These three waypoints are arranged in a triangular pattern. A simple switching algorithms tells the sector-of-sight controller which waypoint to aim for (see also Fossen et al. (2003)). When the profiler is within a circle of acceptance of the current waypoint, the next waypoint is given to the feedback controller by the switching algorithm.

The model and controller were implemented in Matlab/Simulink and represented graphically in a 3D environment using Simulink 3D Animation. A capture of the animation for a simulation run with the clamp mechanism can be seen in Figure 8, where the profiler initial location is at the bottom of the picture, goes in a few dives to the waypoint in the upper left corner, continues to the right to the second waypoint before coming back to its initial location. The blue arrow in the left corner of the picture represents the current direction, while the red bars represent the current sectorof-sight. From this figure, we can see that, depending on the orientation of the mean current, it takes more 


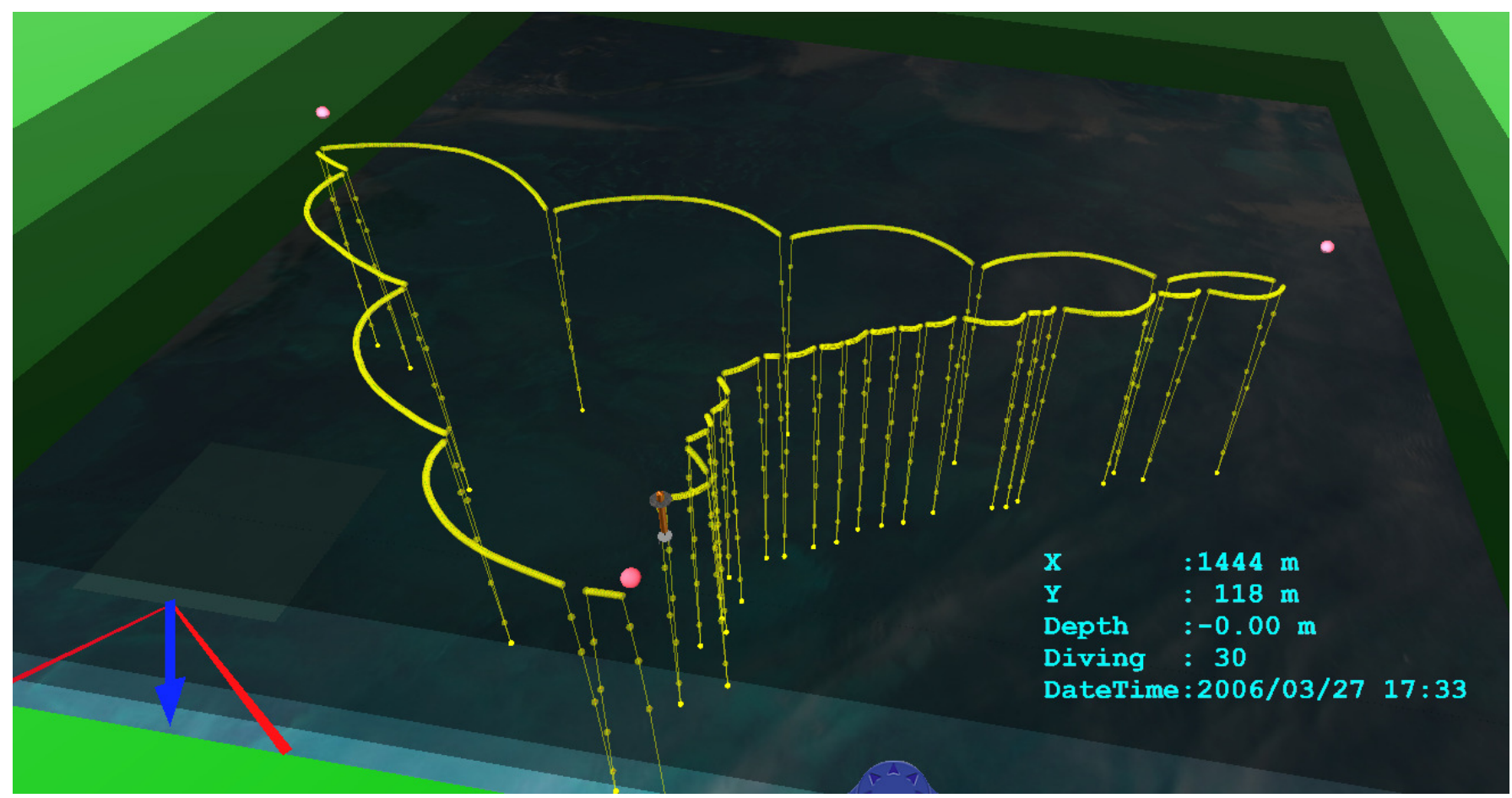

Figure 8: Trajectory of the profiler (clamp mechanism) in a 3-D virtual environment.

or less dives to travel from one waypoint to another.

In Figure 9 is represented a trajectory for the same kind of scenario, this time applied to a profiler model with a weight mechanism. Note the slight drifting of the profiler when parked on the seabed (at depth $-50 \mathrm{~m})$. Obviously, an insufficient friction of the mechanism would induce too much drifting, leading to an inability of the profiler to go against the mean current.

\section{Concluding remarks}

This article proposed feedback control strategies for autonomous Lagrangian profilers, so that a profiler can actively use currents to go to any desired destination, provided the local current structure allows to go in all directions. Despite their simplicity, these strategies were mathematically shown to converge to a neighborhood of the chosen destination, even when this destination is against the mean current direction.

Current research is dedicated to the consideration of more scenarios, in particular in a deep-sea environment, modifications of the proposed algorithms towards a decrease in the number of dives to save further energy, and full-scale experimental testing.

\section{References}

Aeyels, D. and Peuteman, J. A new asymptotic stability criterion for nonlinear time-variant differential equations. IEEE Transactions on Automatic Control, 1998. (7):968-971. doi:10.1109/9.701102.

André, X., Reste, S. L., and Rolon, J.-F. Arvor-C: A coastal autonomous profiling float. Sea Technology, 2010. 51(2):10-13.

Davis, R. E., Webb, D. C., Regier, L. A., and Dufour, J. The Autonomous Lagrangian Circulation Explorer (ALACE). Journal of Atmospheric and Oceanic Technology, 1992. 9:264-285. doi:10.1175/15200426(1992)009<0264:TALCE>2.0.CO;2.

Forward, R. B. and Tankersley, R. A. Selective tidalstream transport of marine animals. Oceanogr. Mar. Biol. Annu. Rev., 2001. 39:305-353.

Fossen, T. I. Marine Control Systems: Guidance, Navigation and Control of Ships, Rigs and Underwater Vehicles. Marine Cybernetics AS, 2002.

Fossen, T. I., Breivik, M., and Skjetne, R. Line-of-sight path following of underactuated marine craft. In Proc. of the IFAC MCMC'03. Girona, Spain, 2003. 


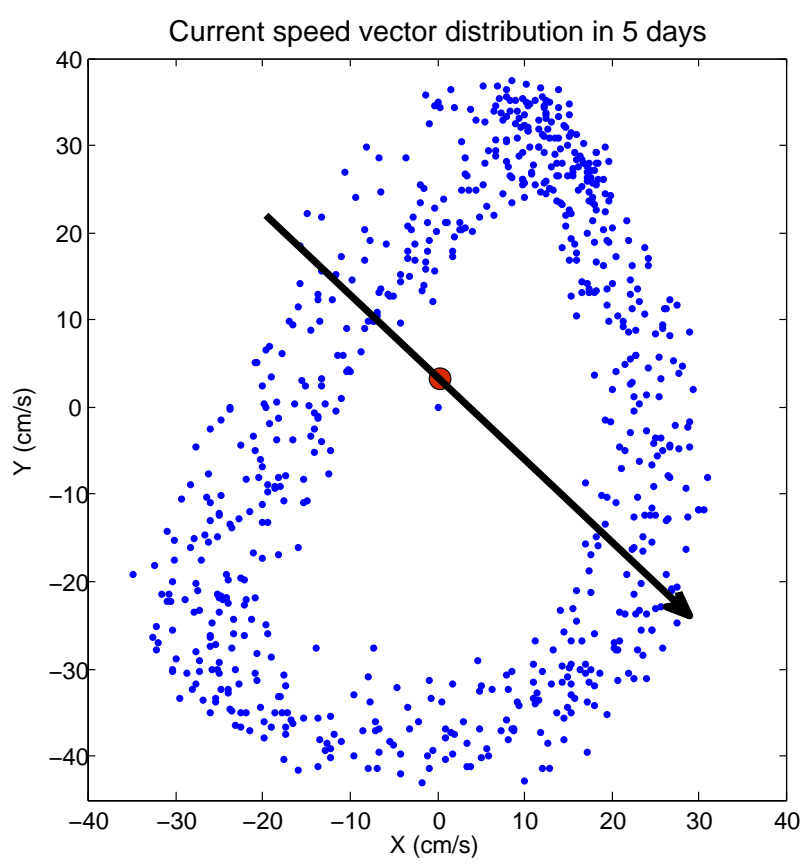

Figure 7: Tidal current speed distribution at location (N54 $59^{\prime} 50^{\prime \prime}, \mathrm{E}^{\circ} 54^{\prime} 30^{\prime \prime}$ ) from $14 / 03 / 2006$ to $19 / 03 / 2006$.

Freeland, H. J., Roemmich, D., Garzoli, S. L., Traon, P.-Y. L., Ravichandran, M., Riser, S., Thierry, V., Wijffels, S., Belbéoch, M., Gould, J., Grant, F., Ignazewski, M., King, B., Klein, B., Mork, K. A., Owens, B., Pouliquen, S., Sterl, A., Suga, T., Suk, M.-S., Sutton, P., Troisi, A., Vélez-Belchi, P. J., and $\mathrm{Xu}, \mathrm{J}$. Argo - a decade of progress. In OceanObs'09. Venice, Italy, 2009.

Gibson, R. N. Go with the flow: tidal migration in marine animals. Hydrobiologia, 2003. 503:153-161. doi:10.1023/B:HYDR.0000008488.33614.62.

Healey, A. J. and Lienard, D. Multivariable slidingmode control for autonomous diving and steering of unmanned underwater vehicles. IEEE Journal of Oceanic Engineering, 1993. (3):327-338. doi:10.1109/JOE.1993.236372.

Hill, A. E. Horizontal zooplankton dispersal by diel vertical migration in $s_{2}$ tidal currents on the northwest european continental shelf. Continental Shelf Research, 1994. 14(5):491-506. doi:10.1016/02784343(94)90100-7.

Jouffroy, J. and Opderbecke, J. Underwater navigation using diffusion-based trajectory observers. IEEE Journal of Oceanic Engineering, 2007. (2):313-326. doi:10.1109/JOE.2006.880392.

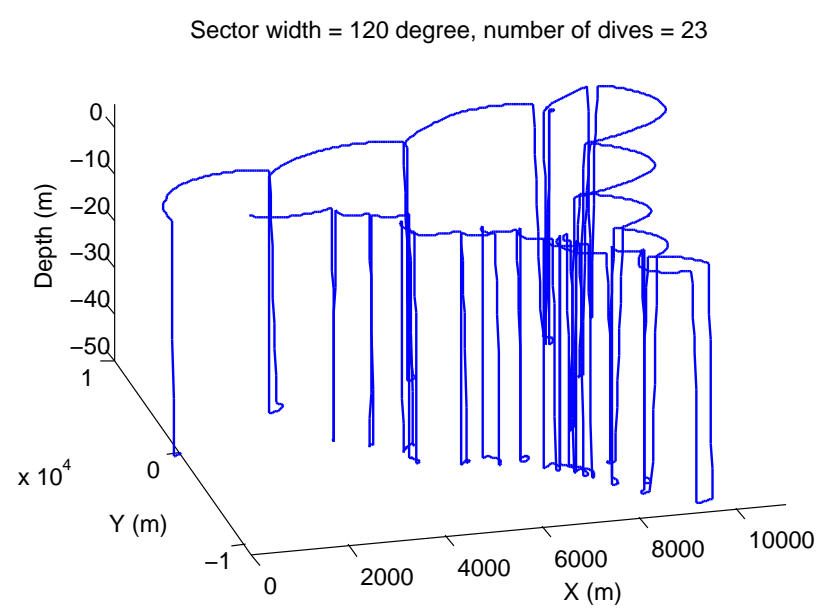

Figure 9: Trajectory of the profiler (weight model mechanism).

Jouffroy, J., Zhou, Q.-Y., and Zielinski, O. Towards selective tidal-stream transport for lagrangian profilers. In MTS/IEEE Oceans'11. Kona, Hawaii, USA, 2011.

Loaec, G., Cortes, N., Menzel, M., and Moliera, J. Provor: a hydrographic profiler based on marvor technology. In IEEE Oceans'98. Nice, France, 1998.

Roemmich, D., Riser, S., Davis, R., and Desaubies, Y. Autonomous profiling floats: workhorse for broad-scale ocean observations. Marine Technology Society Journal, 2004. 38(1):31-39. doi:10.4031/002533204787522802.

Teel, A., Aeyels, D., and Peuteman, J. Semiglobal practical asymptotic stability and averaging. Systems \& Control Letters, 1999. 37:329-334. doi:10.1016/S0167-6911(99)00039-0.

Walters, R. A. A model for tides and currents in the english channel and southern north sea. $A d$ vances in Water Resources, 1987. 10(3):138-148. doi:10.1016/0309-1708(87)90020-0.

Weihs, D. Tidal stream transport as an efficient method for migration. Journal du Conseil International pour l'Exploitation de la Mer, 1978. 38:92-99. doi:10.1093/icesjms/38.1.92.

Welch, J. M. and Forward, R. B. Flood tide transport of blue crab, Callinectes sapidus, postlarvae: behavioral responses to salinity and turbulence. Marine Biology, 1978. 139:911-918. doi:10.1007/s002270100649. 